\title{
Glucosylated Epigallocatechin Gallate (EGCG) Derivatives Combined with EGFR Tyrosine Kinase Inhibitor Overcome Resistance in EGFR T790M Mutant Lung Cancer
}

\author{
Jing Wang ${ }^{1,3 \#}$, Yu-Na Wang ${ }^{1,3}$, Pei-Yuan Sun ${ }^{1,3}$, Cheng-Ting Zi ${ }^{1,2 *}$, Xuan-Jun Wang ${ }^{1,2 *}$, and Jun Sheng ${ }^{1,3 *}$ \\ ${ }^{1}$ Key Laboratory of Pu-er Tea Science, Ministry of Education, China \\ ${ }^{2}$ College of Science, Yunnan Agricultural University, China \\ ${ }^{3}$ College of Food Science and Technology, Yunnan Agricultural University, China \\ \#These authors contributed equally to this work \\ *Corresponding author: Cheng-Ting Zi, Key Laboratory of Pu-er Tea Science, Ministry of Education, College of Science, Yunnan \\ Agricultural University, Kunming, 650201, China
}

Xuan-Jun Wang, Key Laboratory of Pu-er Tea Science, Ministry of Education, College of Science, Yunnan Agricultural University, Kunming, 650201, China

Jun Sheng, Key Laboratory of Pu-er Tea Science, Ministry of Education, College of Food Science and Technology, Yunnan Agricultural University, Kunming, 650201, China

\begin{abstract}
ARTICLE INFO
Received: 幽 May 09, 2019

Published: 閩 May 24, 2019

Citation: Jing Wang, Yu-Na Wang, Pei-Yuan Sun, Cheng-Ting Zi Xuan-Jun Wang, Jun Sheng. Glucosylated Epigallocatechin Gallate (EGCG) Derivatives Combined with EGFR Tyrosine Kinase Inhibitor Overcome Resistance in EGFR T790M Mutant Lung Cancer. Biomed J Sci \& Tech Res 18(2)-2019. BJSTR. MS.ID.003133.
\end{abstract}

Keywords: EGCG; Glycosides; NSCLC; EGFR TKIs; Combination

\begin{abstract}
EGFR tyrosine kinase inhibitors (TKIs) gefitinib and erlotinib are approved treatments for non-small cell lung cancers harboring activating mutations. Unfortunately, treatment with tyrosine kinase inhibitors for a period of time will result in drug resistance, most frequently due to the secondary T790M mutation. Epigallocatechin gallate (EGCG) is the most abundant and bioactive catechin in green tea, it has a strong inhibitory effect on various cancers. We synthesized and identified two glucosylated EGCG derivatives. In this study, we found that the stability and transepithelial transport rate of EGCG derivatives was improved compared with EGCG. Glucosylated EGCG-G2 treatment at different concentrations significantly inhibited the cell growth of EGFR L858R/T790M mutant cells. It was found in vitro that EGCG-G2 combined with gefitinib significantly inhibited the proliferation. More importantly, EGCG-G2 combined with gefitinib downregulate EGFR phosphorylate in NCI-H1975 cells. Taken together, our study suggests that combination of EGFR-TKI and EGCG derivatives maybe a potential treatment strategy to conquer drug resistance for acquired resistant NSCLC.
\end{abstract}

Abbreviations: EGFR: Epidermal Growth Factor Receptor; EGCG: (-)-Epigallocatechin3-Gallate; SDS: Sodium Dodecyl Sulfate; EGFR-TKIs: Epidermal Growth Factor Receptor Tyrosine Kinase Inhibitors; TEER: Trans Epithelial Electric Resistance; Papp: Apparent Permeability Coefficients

\section{Introduction}

Lung cancer is one of the most common malignancies in the world, non-small cell lung cancer (NSCLC) accounting for $85 \%$ of all cases of lung cancers [1]. Epidermal growth factor receptor (EGFR) is an important target in the targeted therapy of NSCLC [2]. It is lo cated in the cell surface and overexpressed in about $50 \%$ of NSCLC patients. This leads to abnormal activation of anti-apoptotic signaling pathway and abnormal cell proliferation [3]. The inhibition of EGFR signaling by tyrosine kinase inhibitors or monoclonal anti- 
bodies plays a key role in NSCLC treatment [4-5]. Epidermal growth factor receptor tyrosine kinase inhibitors (EGFR-TKIs), such as gefitinib and erlotinib have exhibited remarkable therapeutic effects against NSCLC with exon 19 deletions and L858R point activating mutations in EGFR, but resistance arises rapidly in all patients after varying periods of time [6-7]. Most frequently due to the secondary T790M mutation within the ATP-site of the receptor, which has been detected in 50\% of NSCLC cases with acquired resistance [8]. Due to the limited treatment options available for individuals with advanced lung cancer, the novel strategies to conquer drug resistance is an emergency to prolong overall survival time of NSCLC patients.

Epigallocatechin gallate (EGCG) is the most abundant component and most active phenolic constituent of green tea catechins, has been extensively studied in various cancers such as liver cancer, lung cancer, breast cancer, throat cancer, prostate cancer and bladder cancer [9-10]. EGCG also inhibited EGFR tyrosine kinase activity in A431 human epidermal carcinoma cells [11]. EGCG inhibits the phosphorylation of EGFR and is considered to be an important anti-tumor mechanism of EGCG. In our previous study, two novel EGCG glycosides (EGCG-G1 and EGCG-G2) were chemo selectively synthesized by a chemical modification strategy. The EGCG glycosides are more stable than EGCG in aqueous solutions and the EGCG glycosides exhibited increased water solubility: EGCG-G2 and EGCG-G1 were 15 and 31 times as soluble EGCG, respectively [12]. In this study, we further studies regarding the biological activity and bioavailability of EGCG glycosides. In the current study, we explored the antitumor effect of EGCG glycosides on NCI-H1975 which is an acquired EGFR-TKI resistant cell line with T790M and L858R mutations. We investigate whether EGCG glycosides combined treatment with EGFR TKIS could synergistically inhibit NCI-H1975 cell growth by through suppressing phosphorylation of EGFR. Meanwhile, we established a caco-2 cell monolayer model and simulated the absorption of EGCG and EGCG glycosides in the intestinal tract. Our current studies suggest that the combination of EGCG glycosides and EGFR TKIs may provide a potential strategy for NSCLC.

\section{Materials and Methods}

\section{Materials}

Glycosylated EGCG derivatives EGCG-G1 and EGCG-G2 were synthesize in our previous research, Dimethylthiazol-2-yl)-2,5diphenyltetrazolium bromide (MTT) purchased from Sigma-Aldrich (St. Louis, MO, United States). Epigallocatechin-3-gallate (EGCG) and EGFR TKIs were obtained from Sigma-Aldrich (St. Louis, MO, United States), Antibodies against phospho-EGFR (Tyr1068) and EGFR were obtained from Abcom (Lake Placid, NY, USA). The anti$\beta$-tubulin antibody was obtained from Proteintech (Rosemont, IL, United States). Anti-mouse IgG peroxidase-linked whole antibodies and anti-rabbit IgG peroxidase-linked species-specific whole antibodies were from Thermo Fisher Scientific (Waltham, MA, United States).

\section{Cell Cultures}

The human NSCLC cell lines NCI-H1975 and the human colorectal adenocarcinoma cell line Caco-2, were obtained from the American Type Culture Collection (ATCC). The NCI-H1975 cell line with T790M (exon 20) and L858R (exon 21) point mutations was cultured in RPMI-1640 medium (Thermo Fisher Scientific, Pittsburgh, PA, USA) supplemented with $50 \mathrm{IU} / \mathrm{mL}$ of penicillin, $1 \%$ of streptomycin (Solarbio, Beijing, China), and $10 \%$ of fetal bovine serum (HyClone, CA, USA) at $37^{\circ} \mathrm{C}$ in a humidified $5 \% \mathrm{CO}_{2}$ incubator. The human colorectal adenocarcinoma cell line Caco-2 was cultured in DMEM high-glucose medium (Thermo Fisher Scientific, Pittsburgh, PA, USA) in the presence of $10 \% \mathrm{FBS}, 50 \mathrm{IU} / \mathrm{mL}$ of penicillin, and $1 \%$ of streptomycin and maintained at $37^{\circ} \mathrm{C}$ in a humidified atmosphere with $5 \% \mathrm{CO}_{2}$.

\section{MTT Assay}

Cell viability of NSCLC cell line was evaluated by MTT assay. NCI-H1975 cells were seeded in 96-well plates at a density of $2 \times$ 104 cells/well overnight and then treated with EGCG derivatives (0, $10,20,40,60,80$ or $150 \mu \mathrm{M})$, EGFR TKIs alone $(1 \mu \mathrm{M})$, or a combination treatment of EGFR TKIs $(1 \mu \mathrm{M})$ and EGCG derivatives $(30 \mu \mathrm{M})$ for 48 hours. Then added $20 \mu \mathrm{L}$ MTT $(5 \mathrm{mg} / \mathrm{mL}$ in phosphate buffered saline) to the each well, and the plates were incubated for 4 $\mathrm{h}$ at $37^{\circ} \mathrm{C}$. The culture medium was aspirated and $150 \mu \mathrm{Ldimethyl}$ sulfoxide was added to dissolve the formazan crystals. The optical density (OD) was measured at $492 \mathrm{~nm}$ using a microplate reader. The percentage of inhibition was calculated as follows:

$$
\text { Inhibition ratio }(I R, \%)=(1-O D(\text { sample }) / O D(\text { control })) \times 100 \% \text {. }
$$

\section{Western Blot Analysis}

After treatment, NCI-H1975 cells were lysed in RIPA buffer containing PMSF (protease and phosphatase inhibitors) and quantified via BCA protein assay. Proteins separated on 8\% SDSPAGE electrophoresis and then blotted onto polyvinyl difluoride membranes. After the membranes were blocked with BSA for 1 hour, the expression of various proteins was detected using primary $(1 / 1000)$ and secondary antibodies conjugated with horseradish peroxidase (1/800).The Pro-light HRP Chemiluminescent Kit (Tiangen Biotech, Beijing, China) and FuorChem E System (Protein Simple, Santa Clara, CA, United States) were used to detect immunoreactive proteins.

\section{Preparation of Caco-2 Monolayers}

Caco-2 cells were grown as epithelial monolayers by seeding cells onto fibrillar collagen-coated polyethylene terephthalate (PET) Corning transwell inserts $\left(1.12 \mathrm{~cm}^{2}\right)$ with a pore size of $0.4 \mu \mathrm{m}$ at a density of $4.0 \times 10^{5}$ cells/insert and incubated at $37^{\circ} \mathrm{C}$ in an atmosphere of $5 \% \mathrm{CO}_{2}$. The medium was replaced every 2 days with $0.5 \mathrm{~mL}$ medium in the apical (AP) side and $1.5 \mathrm{~mL}$ in the basolateral (BL) side over a period of 21 days. The integrity of the cell monolayer was assessed by testing transepithelial electrical resistance (TEER). 


\section{Transepithelial Electrical Resistance (TEER) Measure- ments}

The integrity of Caco-2 monolayer was determined by measuring the transepithelial electrical resistance (TEER) of the cell monolayer grown on filter supports using an Epithelial Volt Ohm Meter (EVOM) ${ }^{2}$ and electrode set (World Precision Instruments; Sarasota, FL) [13]. To measure TEER, the chopstick-like electrodes were thoroughly rinsed with $70 \%$ ethanol before the start of each experiment, and also after each measurement from each individual well. The electrodes were immersed in a way that shorter electrode was in the insert and longer electrode in the outer well. When measuring the TEER values of monolayers, the electrodes were carefully immersed in the culture medium of the transwell system. The TEER values for each monolayer were monitored every 2 days until the TEER values exceeded $350 \Omega \cdot \mathrm{cm}^{2}$. TEER $\left(\Omega \cdot \mathrm{cm}^{2}\right)=($ measured resistance value - the resistance value of the blank transwell $) \times$ the membrane surface area of transwell.

\section{EGCG And EGCG Derivatives Transport Assay}

Before initiating the transport experiments, the monolayers were washed twice with warm $\left(37^{\circ} \mathrm{C}\right)$ HBSS. The incubation buffer on both sides of the monolayers was then removed by aspiration. EGCG and EGCG-G2 in $0.5 \mathrm{~mL}$ HBSS was added to the AP side or $1.5 \mathrm{~mL}$ added to the BL side. After each incubation time of 0.5 and 1 hour at $37^{\circ} \mathrm{C}$, an aliquot $(0.1 \mathrm{~mL})$ was collected from the receiving chamber for LC-MS analysis.

\section{The Apparent Permeability (Papp)}

The apparent permeability coefficients (Papp) was expressed in $\mathrm{cm} /$ second and was calculated by,

$$
\text { P"app"="dQ" /"dt" ×1/(A·C0) }
$$

Where dQ/dt is the amount of solutes transported across the Caco-2 barrier in time $\mathrm{dt}, \mathrm{C}_{0}$ is the initial drug concentrate, and $\mathrm{A}$ is the cross-sectional area of the epithelium in contact with apical solution.

\section{Results and Discussion}

The Synthesis of EGCG-G1 (2) and EGCG-G2 (3) EGCG glucoside derivatives ( 2 and 3 ) were prepared starting from EGCG, we have used D-glucose and epigallocatechin gallate (EGCG) in a chemical modification strategy to synthesize two novel glycosylated EGCG derivatives EGCG-G1 and EGCG-G2 and characterized their structures. EGCG-G1 (2) contains one $\beta$-glucosidic linkage on the D ring of EGCG, and EGCG-G2 (3) contains two $\beta$-glucosidic linkages on the $\mathrm{C}$ and $\mathrm{D}$ rings, respectively [12].

\section{Effects of EGCG and EGCG Derivatives on NSCLC NCI-H1975 Cells}

Human NSCLC cell line NCI-H1975 (mutant EGFR with L858R and T790M, lose sensitivity to EGFR TKIs) was used in this study [14]. Figure 1 We investigated the cytotoxicity of EGCG and EGCG derivatives on NCI-H1975 cells by 3-(4,5-dimethylthiazol-2-yl)-2,5-diphenyltetrazolium bromide (MTT) assay. As shown in Figure 2A, EGCG-G2 significantly inhibited the cell growth of NCI-H1975 cells compared with EGCG and EGCG-G1. Meanwhile, we test the stability of EGCG and its derivatives according to the amount of hydrogen peroxide $\left(\mathrm{H}_{2} \mathrm{O}_{2}\right)$ which is a by-product of biological oxidation processes. Consistent with the experiments in our previous paper, EGCG glycosides EGCG-G1 and EGCG-G2 are more stable than EGCG.

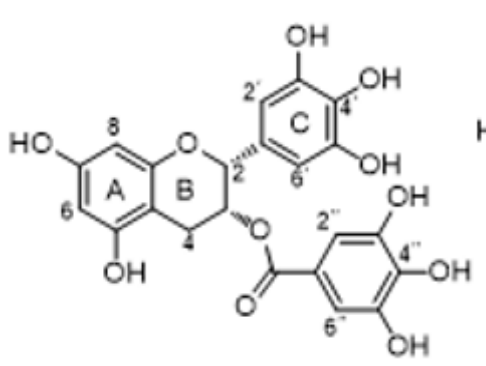

EGCG 1<smiles></smiles>

EGCG-G1 2

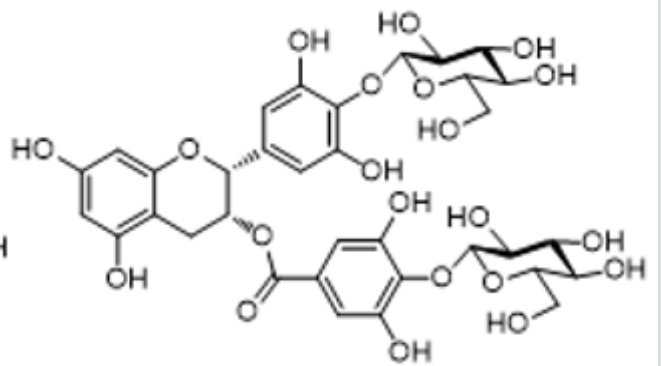

EGCG-G2 3

Figure 1: Structures of EGCG (1), and its glucoside derivatives $(2,3)$.

\section{Effect of EGCG Derivatives in Combination with EGFR TKIs on the Proliferation of NCI-H1975 Cells}

To investigate the effect of EGCG derivatives alone and in combination with EGFR TKIs on the growth of NCI-H1975 cells, we treated the NCI-H1975 cells with varying EGFR TKIs (gefitinib; erlotinib; lapatinib; icotinib; mubritinib and vandetanib) by cell growth assay (Figure 3A-3F). It has been reported that almost all tumors acquire resistance to EGFR-TKIs after varying periods of treatment time $[8,15]$. As shown in Figure 2A, gefitinib combinated with EGCG-G2 resulted in more pronounced growth inhibition than gefitinib and EGCG-G2 alone. 
A

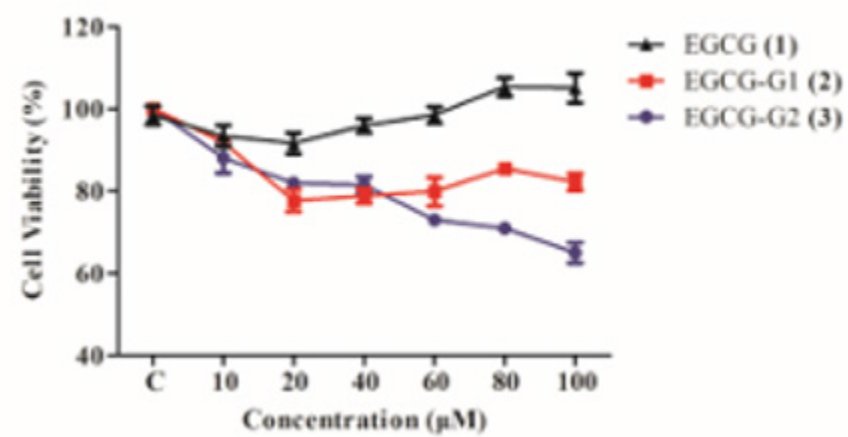

B

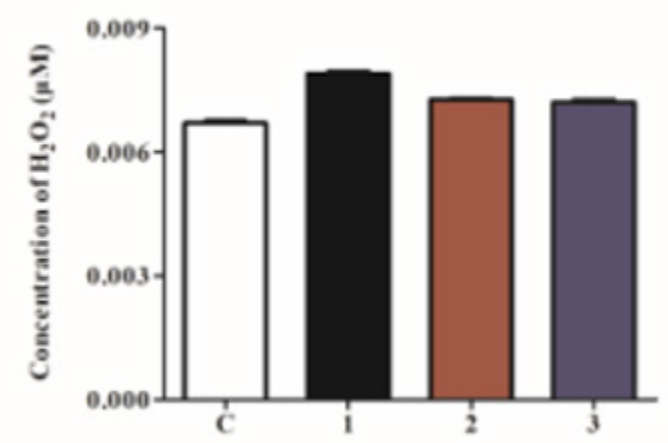

Figure 2: Effect of EGCG and EGCG derivatives on the proliferation of NSCLC cells. A. NCI-H1975 cells were treated with increasing doses of EGCG (1), EGCG-G1 (2) and EGCG-G2 (3) at the indicated concentrations for 48 hours. Cell proliferation was measured by MTT assay and expressed as the proliferation rate. B. $\mathrm{H}_{2} \mathrm{O}_{2}$ concentrations of EGCG (1), EGCG-G1 (2) and EGCG-G2 (3), tested using a $\mathrm{H}_{2} \mathrm{O}_{2}$ Quantitative Assay Kit (Water-Compatible).

A

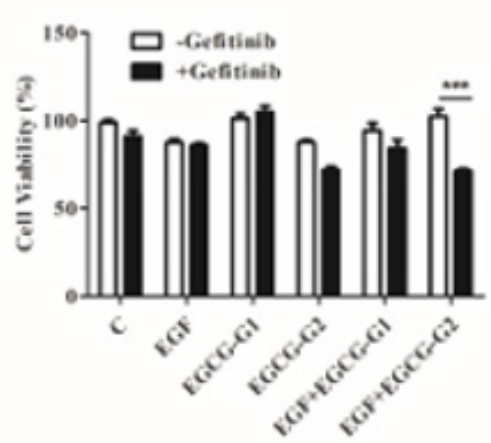

D

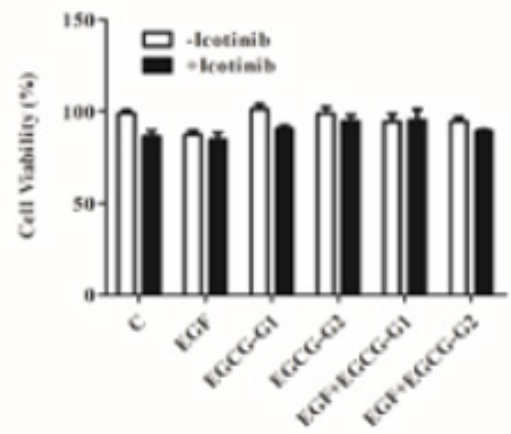

B

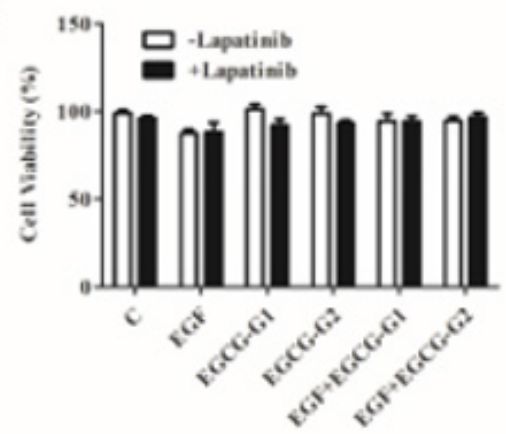

E

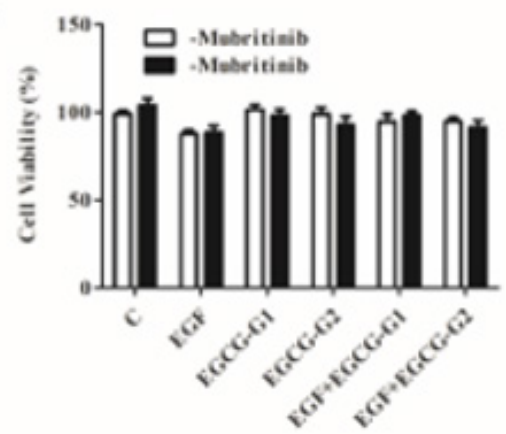

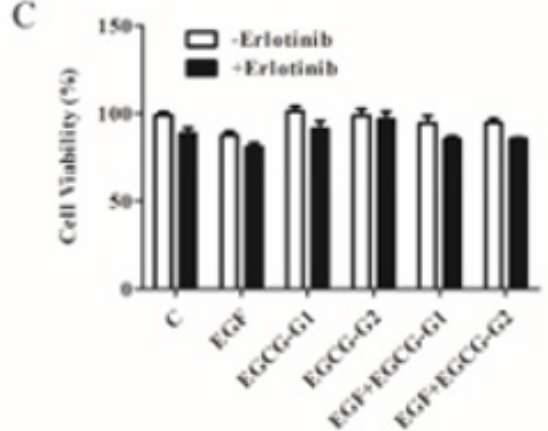

$\mathbf{F}$

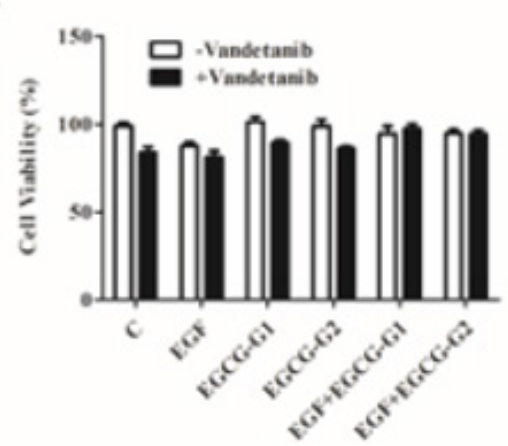

Figure 3: Effect of EGFR TKIs, EGCG derivatives or a combination of the two drugs on the proliferation of NCI-H1975 cells. A-F. gefitinib; erlotinib; lapatinib; icotinib; mubritinib and vandetanib $(1 \mu \mathrm{M})$ combinated with EGCG derivatives (EGCG-G1 and EGCG-G2) $(30 \mu \mathrm{M})$ with or withnot $10 \mathrm{ng} / \mathrm{mL}$ EGF. Data represent the average of three independent experiments (mean \pm SEM, $\mathrm{p}<0.001) .{ }^{* * *} \mathrm{p}<0.001$.

\section{Effect Of EGCG-G2 in Combination with Gefitinib on EGFR}

\section{Signaling}

The EGFR signal pathway is a crucial target in NSCLC treatment [7]. To characterize whether the growth inhibition induced by EGCG-G2 and gefitinib might involve EGFR signaling, we examined the phosphorylation levels of the members of the Her (ErbB) family of receptor tyrosine kinases, which includes EGFR (ErbB1), Her2 (ErbB2/Neu), Her3 (ErbB3), and Her4 (ErbB4). NCI-H1975 cells were treated with EGCG-G2 and gefitinib alone or combination of two drugs for $12 \mathrm{~h}$. NCI-H1975 cells were stimulated with $10 \mathrm{ng} / \mathrm{mL}$ EGF for 10 minutes. As shown in Figure 4, in combination with gefitinib, EGGC-G2 inhibited the phosphorylation of EGFR family more significantly than the alone. 


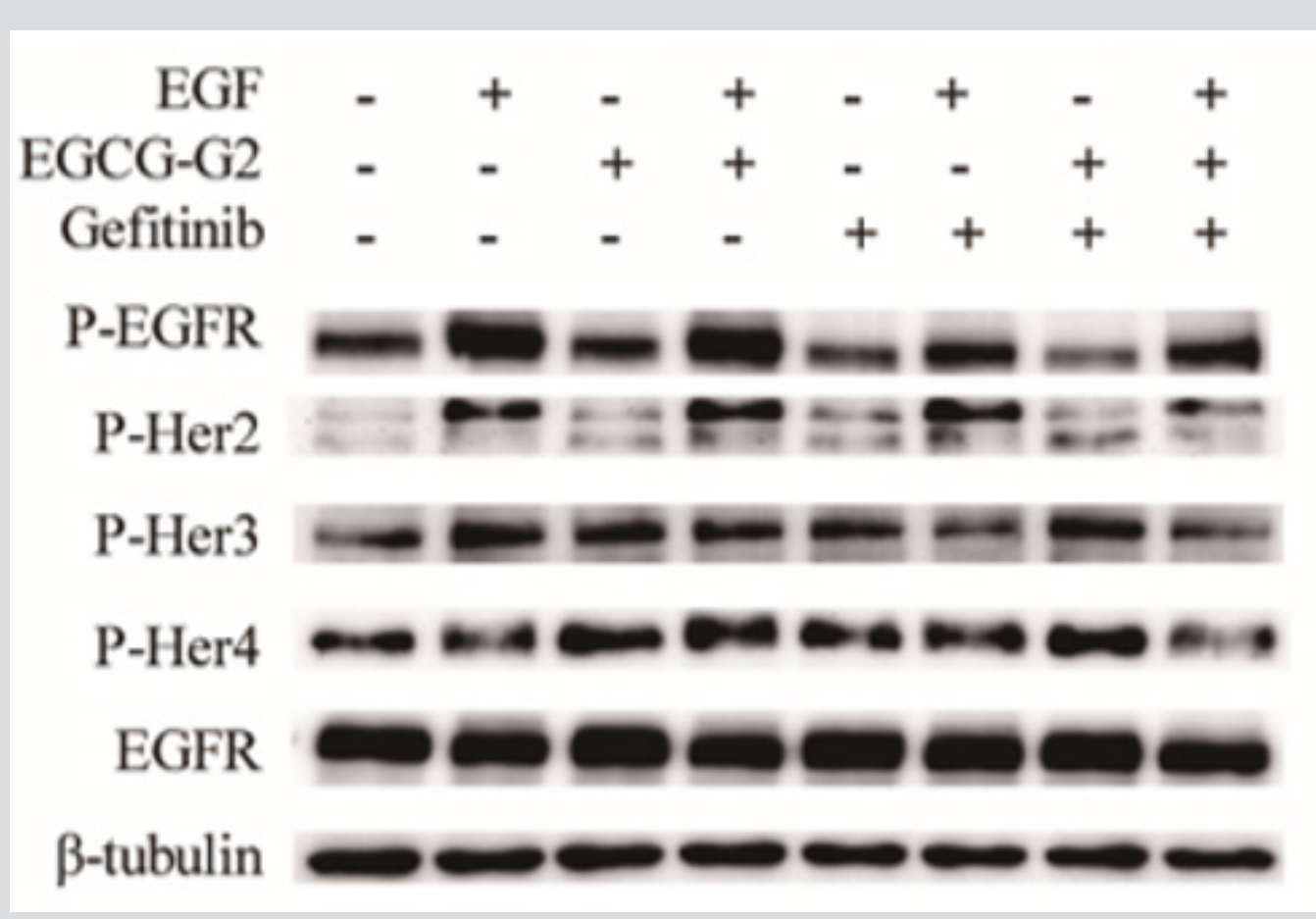

Figure 4: Combination of EGCG-G2 and grlotinib has synergized inhibitory effects on the phosphorylation the four members of the Her (ErbB) family in H1975 cells. NCI-H1975 cells were treated with EGCG-G2 (30 $\mu \mathrm{M})$, gefitinib $(1 \mu \mathrm{M})$ or EGCG-G2 plus gefitinib, after 12 hours, NCI-H1975 cells were stimulated with or withnot $10 \mathrm{ng} / \mathrm{mL}$ EGF for 5 minutes. The expression levels of the proteins were determined by western blotting with $\beta$-tubulin as the loading control.

\section{Transport of EGCG and EGCG-G2 Across Caco-2 Cell Monolayers}

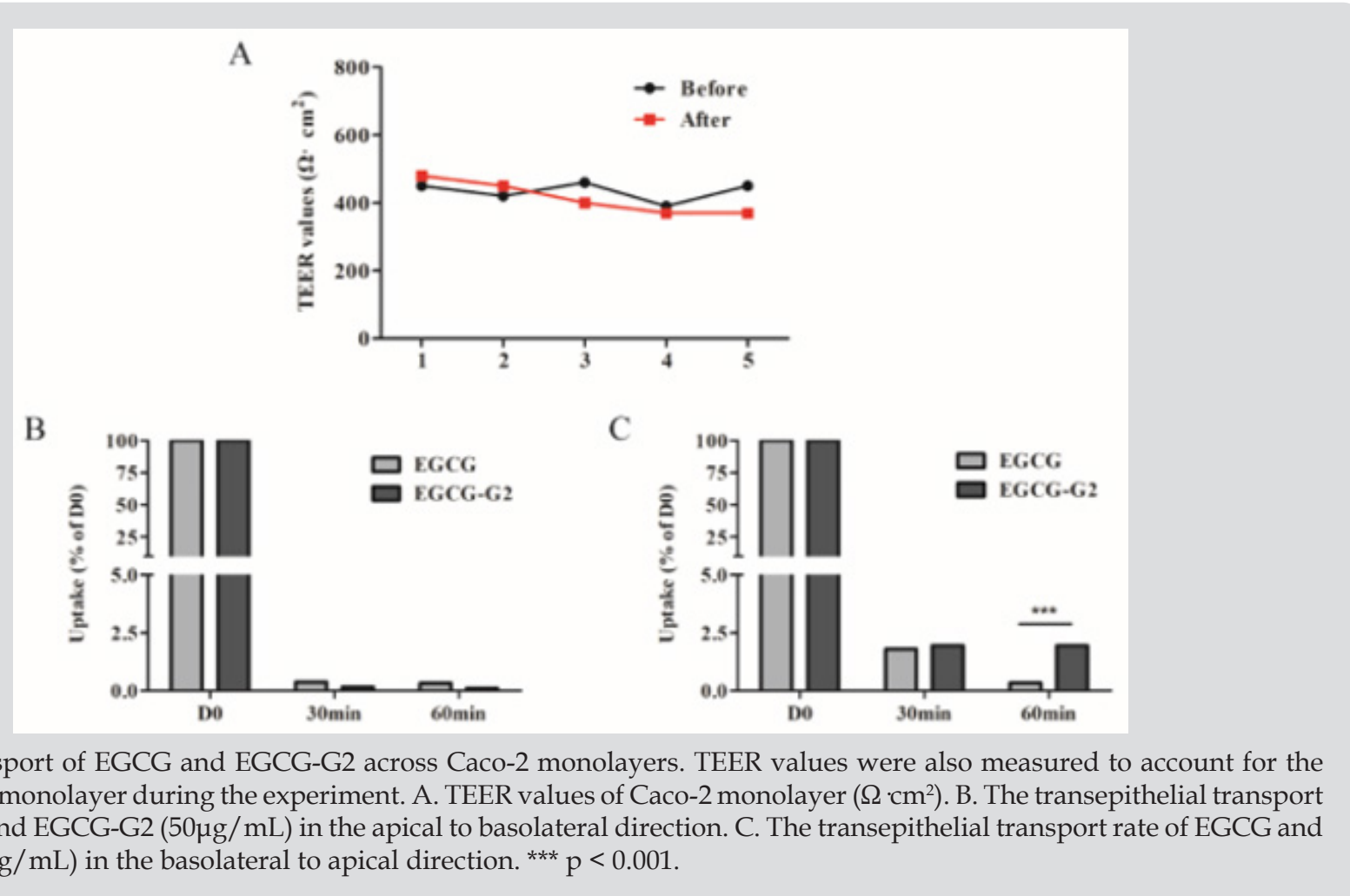

According to the TEER value it was concluded that the integrity and tightness of epithelial cell monolayers were maintained after 21 days culturing [16]. To ensure monolayer integrity during the assay, all wells were monitored TEER values before and after the experiments. The TEER values did not exhibit a drop during the experiment, indicating that the cell monolayer was intact and the transport of EGCG and EGCG-G2 did not damage the monolayer (Figure 5A). The transported amounts of EGCG and EGCG-G2 were 
assessed for both the apical to basolateral $(\mathrm{AB})$ and basolateral to apical (BA) directions. As shown in Figure 5B and 5C, the amounts of EGCG-G2 transported in the acceptor chamber were higher in the $B A$ direction than in the $\mathrm{AB}$, and the uptake of EGCG-G2 in the BA direction was 5.7-fold higher than the corresponding values of EGCG at $60 \mathrm{~min}$. The apparent permeability coefficients (Papp) values of EGCG-G2 in the BA direction were $7.55 \times 10-7 \mathrm{~cm} / \mathrm{s}$ and $3.2 \times 10-7$ $\mathrm{cm} / \mathrm{s}$ were significantly higher than the $\mathrm{AB}$ direction.

\section{Conclusion}

In conclusion, glucosylated epigallocatechin gallate (EGCG) derivatives have improved stability and absorption compared to EGCG. EGCG-G2 has shown significantly inhibitory effect on EGFR L858R/T790M mutant NSCLC cells. In addition, EGCG-G2 combinated with gefitinib markedly inhibited EGF-induced EGFR phosphorylation Table 1. EGCG-G2, either alone or in combination with gefitinib has the potential in EGFR-TKI- resistant NSCLC treatment.

Table 1: Papp values for EGCG and EGCG-G2 from the apical to the basolateral chamber and vice versa over different times.

\begin{tabular}{|c|c|c|c|c|}
\hline & \multicolumn{4}{|c|}{ Papp $\left(\mathbf{1 0}^{-\mathbf{7}} \mathbf{~ c m} / \mathbf{s}\right)$} \\
\hline & $\mathbf{3 0} \mathbf{m i n}(\mathbf{A} \rightarrow \mathbf{B})$ & $\mathbf{3 0} \mathbf{m i n}(\mathbf{B} \rightarrow \mathbf{A})$ & $\mathbf{6 0} \mathbf{m i n}(\mathbf{A} \rightarrow \mathbf{B})$ & $\mathbf{6 0} \mathbf{m i n}(\mathbf{B} \rightarrow \mathbf{A})$ \\
\hline EGCG & 0.28 & 1.37 & 0.24 & 0.25 \\
\hline EGCG-G2 & 0.65 & 7.55 & 0.19 & 3.2 \\
\hline
\end{tabular}

Data are reported as mean \pm SEM $(n=3)$.

\section{Acknowledgement}

This research was supported by grants from the National Natural Science Foundation of China (No. 21602196); the Yunnan Provincial Science and Technology Department (Nos. 2017FD084 and 2017FG001-046); Yunnan Agricultural University Natural Science Foundation for Young Scientists and Postgraduates (Nos. 2015ZR08 and 2016ykc53) and Academic Award for Up-andcoming Doctoral Candidates of Yunnan Province (A2008057).

\section{References}

1. Hodkinson PS, Mackinnon A, Sethi T (2008) Targeting Growth Factors in Lung Cancer. Chest 133(5): 1209-1216.

2. Pignon JP, Tribodet H, Scagliotti GV, Douillard JY, Shepherd FA, et al. (2008) Lung Adjuvant Cisplatin Evaluation: A Pooled Analysis by the LACE Collaborative Group. J Clin Oncol 26(21): 3552-3559.

3. Wangaritalbot J, Hopperborge E (2013) Drug Resistance Mechanisms in Non-Small Cell Lung Carcinoma. J Can Res Updates 2(4): 265-282.

4. Marmor MD, Skaria KB, Yarden Y (2004) Signal transduction and oncogenesis by ErbB/HER receptors. Int J Radiat Oncol Biol Phys 58(3): 903-913.

5. Langer CJ (2004) Emerging role of epidermal growth factor receptor inhibition in therapy for advanced malignancy: focus on NSCLC. Int J Radiat Oncol Biol Phys 58(3): 991-1002.

6. Inoue A, Nukiwa T (2005) Gene Mutations in Lung Cancer: Promising Predictive Factors for the Success of Molecular Therapy. Plos Med 2(1): e13.

7. Liu TC, Jin X, Wang Y (2017) Role of epidermal growth factor receptor in lung cancer and targeted therapies. Am J Cancer Res 7(2): 187-202.

\section{ISSN: 2574-1241}

DOI: 10.26717/BJSTR.2019.18.003133

Cheng-Ting Z, Xuan-Jun W, Jun Sheng. Biomed J Sci \& Tech Res

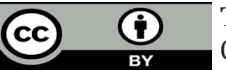

This work is licensed under Creative

Commons Attribution 4.0 License

Submission Link: https://biomedres.us/submit-manuscript.php
8. Sequist LV, Soria JC, Goldman JW, Wakelee HA, Gadgeel SM, et al. (2015) Rociletinib in EGFR-Mutated Non-Small-Cell Lung Cancer. N Engl J Med 372(18): 1700-1709.

9. Zhang X, Zhang H, Tighiouart M, Lee JE, Shin HJ, et al. (2008) Synergistic inhibition of head and neck tumor growth by green tea (-)-epigallocatechin-3-gallate and EGFR tyrosine kinase inhibitor. Int J Cancer 123(5): 1005-1014.

10. Wei R, Mao L, Xu P, Zheng X, Hackman RM, et al. (2018) Suppressing glucose metabolism with epigallocatechin-3-gallate (EGCG) reduces breast cancer cell growth in preclinical models. Food Funct 9(11): 56825696.

11. Ma YC, Li C, Gao F, Xu Y, Jiang ZB, et al. (2013) Epigallocatechin gallate inhibits the growth of human lung cancer by directly targeting the EGFR signaling pathway. Oncol Rep 31(3): 1343-1349.

12. Zhang X, Wang J, Hu JM, Huang YW, Wu XY, et al. (2016) Synthesis and Biological Testing of Novel Glucosylated Epigallocatechin Gallate (EGCG) Derivatives. Molecules 21(5): 620.

13. Song Q, Li D, Zhou Y, Yang J, Yang, W, et al. (2014) Enhanced uptake and transport of $(+)$-catechin and (-)-epigallocatechin gallate in niosomal formulation by human intestinal Caco-2 cells. Int J Nanomedicine 2014: 2157-2165.

14. Pao W, Miller VA (2005) Epidermal growth factor receptor mutations, small-molecule kinase inhibitors, and non-small-cell lung cancer: current knowledge and future directions. J Clin Oncol 23(11): 25562568.

15. Cross DA, Ashton SE, Ghiorghiu S, Eberlein C, Nebhan CA, et al. (2014) AZD9291, an Irreversible EGFR TKI, Overcomes T790M-Mediated Resistance to EGFR Inhibitors in Lung Cancer. Cancer Discov 4(9): 10461061.

16. Kauffman AL, Gyurdieva AV, Mabus JR, Ferguson C, Yan Z, et al. (2013) Alternative functional in vitro models of human intestinal epithelia. Front Pharmacol 4: 79.

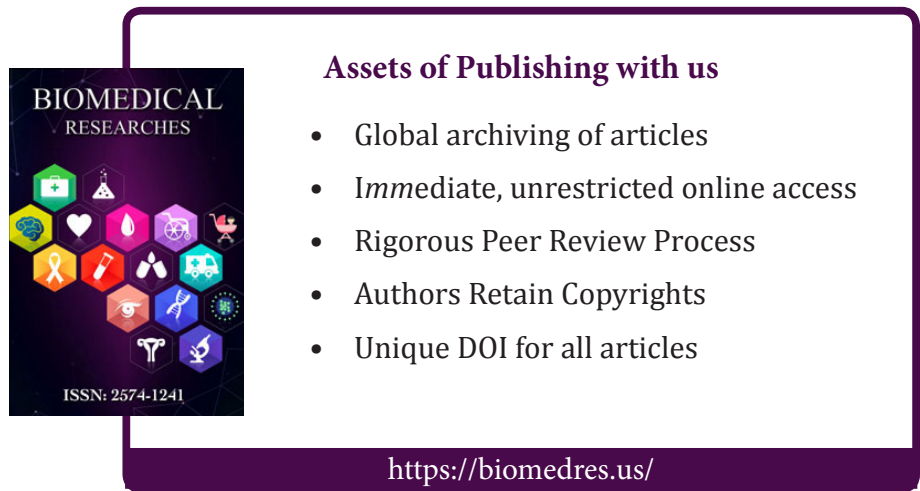

